\title{
NORM PRESERVING EXTENSIONS OF LINEAR TRANSFORMATIONS ON HILBERT SPACES
}

\author{
MICHAEL G. CRANDALL ${ }^{1}$
}

Introduction. Let $H$ be a Hilbert space and let $D$ be a closed proper subspace of $H$. Let $J_{0}$ be a linear contraction on $D$ to $H$. The problem of characterizing the contractions on all of $H$ which extend $J_{0}$ is directly related to the extension problems for unbounded transformations posed and treated by M. G. Krein [2] and R. S. Phillips [3]. In $\$ 1$ of this paper we establish the following solution of this problem: Let $P$ be the orthogonal projection of $H$ on $D$ and $J_{1}=J_{0} P$. The contractions on all of $H$ which extend $J_{0}$ are precisely the transformations of the form $J_{1}+\left(I-J_{1} J_{1}^{*}\right)^{1 / 2} B$, where $B$ is any contraction which annihilates $D$. $\S 2$ is a short outline of the relationship of this result to the extension problem for dissipative transformations treated by Phillips in [3] and [4]. $\$ 3$ contains some remarks concerning the case where $J_{0}$ is symmetric and one asks for the selfadjoint contractions which extend $J_{0}$, which is essentially the situation treated by Krein [2]. In particular, we obtain the Krein extension of a symmetric contraction to a selfadjoint contraction by, we feel, a less elegant but more illuminating argument than Krein's original one.

1. The representation theorem. All transformations considered here are linear. The domain, range and null space of a transformation $L$ will be denoted by $D(L), R(L)$, and $N(L)$ respectively. Our goal is to prove the following theorem:

Theorem I. Let $H$ be a Hilbert space. Let $D$ be a closed proper subspace of $H$ and let $J_{0}$ be a contraction on $D$ to $H$. Let $P$ be the orthogonal projection of $H$ on $D$ and set

$$
J_{1}=J_{0} P .
$$

Then the contractions $J$ on $H$ to $H$ which extend $J_{0}$ are precisely the transformations of the form

$$
J=J_{1}+\left(I-J_{1} J_{1}^{*}\right)^{1 / 2} B
$$

where $B$ is any contraction on $H$ which annihilates $D$.

REMARK. The correspondence (1.2) between contractions $J$ which

Presented to the Society, June 29, 1968; received by the editors May 17, 1968.

1 This report is supported in part by the National Science Foundation Grant GP 7458. 
extend $J_{0}$ and contractions $B$ which annihilate $D$ becomes biunique if we further require that $R(B) \subset[R(T)]$, where we have set

$$
T=\left(I-J_{1} J_{1}^{*}\right)^{1 / 2}
$$

and $[R(T)]$ denotes the closure of $R(T)$.

Proof of Theorem I. For convenience we set

(1.4) $E\left(J_{0}\right)=\left\{J: J\right.$ is a contraction on all of $B$ and $J$ extends $\left.J_{0}\right\}$.

Let $J \in E\left(J_{0}\right)$. Clearly then $J P=J_{1}=J_{0} P$ and we have

$$
J=J P+J(I-P)=J_{1}+J(I-P) \quad \text { for } J \text { in } E\left(J_{0}\right) .
$$

The decomposition (1.5) shows that every element of $E\left(J_{0}\right)$ has the form $J_{1}+K$ where $K$ is a contraction annihilating $D$. Conversely, any transformation of this form extends $J_{0}$. Hence $E\left(J_{0}\right)$ consists of those sums $J_{1}+K$, where $K$ is a contraction annihilating $D$, which are contractions. The theorem is therefore an immediate consequence of the following lemma.

Lемма I. Let the assumptions of Theorem I be satisfied. Then, if $K$ is a contraction on $H$ which annihilates $D, J_{1}+K$ is a contraction if and only if $K$ has the form $K=T B$, where $T$ is given by (1.3), for some contraction $B$ which annihilates $D$.

Proof of Lemma I. An attempt to prove Lemma I directly would lead to some difficulties, however, passing to the adjoint allows a trivial proof. We first show the sufficiency. Let $B$ be a contraction annihilating $D . J_{1}+T B$ is a contraction if $J_{1}^{*}+B^{*} T$ is a contraction (note that $T$ is selfadjoint). Moreover, $N\left(J_{1}\right) \supset D^{\perp}$ (the orthogonal complement of $D$ in $H$ ) and $N(B) \supset D$, so $R\left(J_{1}^{*}\right)$ and $R\left(B^{*}\right)$ are orthogonal. Hence, since $B^{*}$ is a contraction and since $\|T w\|^{2}=\|w\|^{2}-\left\|J_{1}^{*} w\right\|^{2}$ for $w$ in $H$ by (1.3), where \|\| is the norm in $H$, we have

$$
\begin{aligned}
\left\|\left(J_{1}^{*}+B^{*} T\right) w\right\|^{2} & =\left\|J_{1}^{*} w\right\|^{2}+\left\|B^{*} T w\right\|^{2} \leqq\left\|J_{1}^{*} w\right\|^{2}+\|T w\|^{2} \\
& =\left\|J_{1}^{*} w\right\|^{2}+\|w\|^{2}-\left\|J_{1}^{*} w\right\|^{2}=\|w\|^{2}
\end{aligned}
$$

for $w$ in $H$, and $J_{1}^{*}+B^{*} T$ is a contraction.

Conversely, if $K$ annihilates $D$ and $J_{1}+K$ is a contraction, then $R\left(J_{1}^{*}\right)$ and $R\left(K^{*}\right)$ are orthogonal and $J_{1}^{*}+K^{*}$ is a contraction, so we have

$$
\left\|\left(J_{1}^{*}+K^{*}\right) w\right\|^{2}=\left\|J_{1}^{*} w\right\|^{2}+\left\|K^{*} w\right\|^{2} \leqq\|w\|^{2} \quad \text { for } w \text { in } H .
$$

It follows from (1.6) that

$$
\left\|K^{*} w\right\|^{2} \leqq\|T w\|^{2} \quad \text { for } w \text { in } H .
$$


According to (1.7) the map $L$ on $R(T)$ to $R\left(K^{*}\right)$ defined by $L T w$ $=K^{*} w$ is a contraction. We extend $L$ to $[R(T)]$ by continuity and then to all of $H$ by setting it equal to zero on $R(T)^{\perp}$. Then we have $L T=K^{*}$ and $K=T L^{*}$. Since $R(L) \subset R\left(K^{*}\right) \subset D^{\perp}, N\left(L^{*}\right) \supset D$ and, setting $B=L^{*}, K$ has the form claimed by the lemma. This completes the proof of Lemma I and therefore Theorem I is also established.

REMARK. The proof of Theorem I may easily be adapted to provide a proof of the more general result stated below.

Theorem I'. Let $H_{1}$ and $H_{2}$ be Hilbert spaces. Let $D$ be a closed proper subspace of $H_{1}$ and let $J_{0}$ be a bounded transformation on $D$ to $H_{2}$. Let $P$ be the orthogonal projection of $H_{1}$ on $D$ and set $J_{1}=J_{0} P$. Then the bounded transformations on $H_{1}$ to $H_{2}$ of norm not exceeding $k\left\|J_{0}\right\|$, $k \geqq 1$, which extend $J_{0}$ are precisely the transformations of the form $J_{1}+\left(k^{2}\left\|J_{0}\right\|^{2}-J_{1} J_{1}^{*}\right)^{1 / 2} B$, where $B$ is a contraction on $H_{1}$ to $H_{2}$ which annihilates $D$.

2. The extension problem for dissipative transformations. Let $A$ be a transformation on $D(A) \subset H$ into $H$. $A$ is called dissipative if

$$
(u, A u)+(A u, u) \leqq 0 \quad \text { for } u \text { in } D(A) \text {. }
$$

If $A$ is dissipative and has no proper dissipative extensions, it is called maximal dissipative. We consider the problem of finding all the maximal dissipative transformations which extend a given dissipative $A$. If $A$ is dissipative then, in view of (2.1),

$$
\begin{aligned}
\|(I-A) u\|^{2} & =\|u\|^{2}+\|A u\|^{2}-2 \operatorname{Re}(u, A u) \\
& \geqq\|u\|^{2}+\|A u\|^{2} \geqq\|u\|^{2}+\|A u\|^{2}+2 \operatorname{Re}(u, A u) \\
& =\|(I+A) u\|^{2} \text { for } u \text { in } D(A) .
\end{aligned}
$$

It follows from the first inequality of (2.2) that $I-A$ is one-to-one and that $R(I-A)$ is a closed subspace of $H$ if $A$ is a closed transformation, and from the inequality of the extreme members in (2.2) it follows that the transformation $J_{0}$ defined by

$$
D\left(J_{0}\right)=R(I-A), \quad J_{0}(I-A) u=(I+A) u \text { for } u \text { in } D(A)
$$

is a contraction. $J_{0}$ is called the Cayley transform of $A$. If $D(A)$ is dense in $H$, then there is a one-to-one correspondence between the maximal dissipative extensions of $A$ and the elements of $E\left(J_{0}\right)$, that is the contractions which extend $J_{0}$ to $H$. Given $J$ in $E\left(J_{0}\right)$ the corresponding maximal dissipative extension of $A$ is the dissipative transformation $A_{J}$ whose Cayley transform is $J$ : It is defined by

$$
D\left(A_{J}\right)=R(I+J), \quad A_{J}(I+J) u=(J-I) u \text { for } u \text { in } H .
$$


For the straightforward proof of this see [3]. Theorem I, therefore, allows us to characterize the maximal dissipative extensions of a given dissipative transformation $A$. We proceed with this below.

Let $A$ be a closed densely defined dissipative transformation and let $J_{0}$ be the Cayley transform of $A$. Let $P$ be the orthogonal projection of $H$ on $D\left(J_{0}\right)=R(I-A)$ and let $J_{1}=J_{0} P$. $J_{1}$ is determined by $A$ as follows: $R(I-A)$ is closed and $R(I-A)^{\perp}=N\left(I-A^{*}\right)$. Every element $w$ of $H$ has a unique decomposition into a sum $w=(I-A) u+v$ with $u$ in $D(A)$ and $v$ in $N\left(I-A^{*}\right)$ and we have

$$
J_{1}((I-A) u+v)=(I+A) u \text { for } u \text { in } D(A) \text { and } v \text { in } N\left(I-A^{*}\right) \text {. }
$$

$J_{1}^{*}$ is related in a somewhat similar way to $A^{*}$. Since $N(I-A)=\{0\}$ and $R(I-A)$ is closed, $R\left(I-A^{*}\right)=H$. We claim

$$
J_{1}^{*}\left(I-A^{*}\right) u=P\left(I+A^{*}\right) u \text { for } u \text { in } D\left(A^{*}\right) \text {. }
$$

In view of (2.5), the validity of (2.6) is equivalent to

$$
\begin{aligned}
\left((I+A) u,\left(I-A^{*}\right) w\right) & =\left((I-A) u+v, P\left(I+A^{*}\right) w\right) \\
\text { for } u & \text { in } D(A), w \text { in } D\left(A^{*}\right) \text { and } v \text { in } N\left(I-A^{*}\right),
\end{aligned}
$$

which is true in view of

$$
\begin{aligned}
((I-A) u & \left.+v, P\left(I+A^{*}\right) w\right) \\
& =\left(P((I-A) u+v),\left(I+A^{*}\right) w\right)=\left((I-A) u,\left(I+A^{*}\right) w\right)
\end{aligned}
$$

for $u$ in $D(A), w$ in $D\left(A^{*}\right)$ and $v$ in $N\left(I-A^{*}\right)$.

We have now, as an immediate corollary of Theorem I,

Corollary I. Let $A$ be a closed densely defined dissipative transformation. Then there is a one-to-one correspondence between contractions $B$ on $N\left(I-A^{*}\right)$ to $[R(T)]$ where $T$ is given by (1.3), $J_{1}$ by (2.5) and $J_{1}^{*}$ by (2.6), and the maximal dissipative extensions of $A$. Given a contraction $B$ on $N\left(I-A^{*}\right)$ to $[R(T)]$ the corresponding extension $A_{B}$ of $A$ is defined by

$$
\begin{aligned}
D\left(A_{B}\right) & =D(A)+(I+T B) N\left(I-A^{*}\right), \\
A_{B}(u+(I+T B) v) & =A u+(T B-I) v \\
& \text { for } u \text { in } D(A) \text { and vin } N\left(I-A^{*}\right) .
\end{aligned}
$$

REMARK 1. The main shortcoming of this result with regard to applications lies in the difficulty of computing the action of the transformation $T$ given the transformation $A$. With further restrictions on the transformation $A$ more convenient descriptions of its maximal dissipative extensions can be given. See M. G. Crandall and R. S. Phillips [1]. 
REMARK 2. A more general problem, including the extension problem for dissipative transformations, is treated by Phillips [3], [4] and [5]. In Theorem 5.3 of [4] Phillips gives a solution of this problem, the formulation of which involves completing a certain Hilbert space in a new metric. We remark that Theorem I' may be applied directly to this more general problem, giving a new solution which does not involve any "completions."

3. The symmetric case. We adopt here the notations of $\$ 1$. We now assume that $J_{0}$ is symmetric, i.e.,

$$
\left(J_{0} u, v\right)=\left(u, J_{0} v\right) \quad \text { for } u \text { and } \quad v \text { in } D,
$$

and ask for those $J$ in $E\left(J_{0}\right)$ which are selfadjoint. See Krein [2] for the relationship of this question to the problem of characterizing the semibounded selfadjoint extensions of semibounded symmetric transformations. Let $J_{1}=J_{0} P$ as before. Clearly $J_{0}$ is symmetric if and only if $P J_{1}$ is selfadjoint. Let $J=J_{1}+T B$ be an element of $E\left(J_{0}\right) ; B$ is a contraction annihilating $D$ and $T$ is given by (1.3). It is not easy to characterize those $B$ for which $J$ is selfadjoint, but we can easily characterize those $B$ for which $J^{*}$ extends $J_{0}$, and if $J$ and $J^{*}$ both extend $J_{0}$ then $\left(J+J^{*}\right) / 2$ is a selfadjoint extension of $J_{0}$. We let $P_{1}=(I-P) ; P_{1}$ is the orthogonal projection of $H$ on $D^{\perp}$. Then

$$
\begin{aligned}
\left(J_{1}+T B\right)^{*} & =\left(\left(P+P_{1}\right)\left(J_{1}+T B\right)\left(P+P_{1}\right)\right)^{*} \\
& =P J_{1}+P J_{1}^{*} P_{1}+B^{*} T P+B^{*} T P_{1},
\end{aligned}
$$

because $P J_{1}$ is selfadjoint and $J_{1} P_{1}=B P=0$ by assumption, and it follows that $\left(J_{1}+T B\right)^{*}$ extends $J_{0}$ if and only if

$$
P J_{1}+B^{*} T P=J_{1}
$$

or

$$
B^{*} T P=P_{1} J_{1}
$$

Equation (3.2) determines the action of $B^{*}$ on $[T D]$. As noted below, (3.2) is consistent with the requirement that $B^{*}$ be a contraction and Theorem I can be used to characterize those contractions $B$ for which (3.2) is satisfied, which we leave to the reader. Here we restrict ourselves to noting that if $L$ is defined by

$$
\begin{aligned}
L^{*} T u & =P_{1} J_{1} u & & \text { for } u \text { in } D \\
L^{*} v & =0 & & \text { for } v \text { in }(T D)^{\perp}
\end{aligned}
$$

then $L^{*}$ (and hence $\left.L\right)$ is a contraction for $J_{1}^{*} P=\left(P J_{1}\right)^{*}=P J_{1}$ implies 


$$
\begin{aligned}
\|T u\|^{2} & =\|u\|^{2}-\left\|J_{1}^{*} u\right\|^{2}=\|u\|^{2}-\left\|J_{1}^{*} P u\right\|^{2}=\|u\|^{2}-\left\|P J_{1} u\right\|^{2} \\
& =\|u\|^{2}-\left\|J_{1} u\right\|^{2}+\left\|P_{1} J_{1} u\right\|^{2} \\
& \geqq\left\|P_{1} J_{1} u\right\|^{2} \quad \text { for } u \text { in } D,
\end{aligned}
$$

and $R\left(L^{*}\right) \subset D^{\perp}$ implies $N(L) \supset D$. By the above arguments it follows that $\left(J_{1}+J_{1}^{*}+T L+L^{*} T\right) / 2$ is a selfadjoint contraction which extends $J_{0}$; it is the extension found by Krein [2].

REMARK. If $W$ is a bounded selfadjoint transformation on $H$ and

$$
J_{0} W=\varepsilon W J_{0} \quad \text { for } \varepsilon=1 \text { or } \varepsilon=-1 \text {, }
$$

it was noticed by Phillips [5, Lemma 3.1] that the Krein extension of $J_{0}$ will also satisfy (3.4) in place of $J_{0}$. Our construction makes this obvious, for if (3.4) holds then $W$ commutes with $P$ and $P_{1}$, so $J_{1}$ satisfies (3.4) and therefore $J_{1}^{*}$ satisfies (3.4) and therefore $\left(I-J_{1} J_{1}^{*}\right)^{1 / 2}$ commutes with $W$ and finally the transformations $L^{*}$ and $L$ defined by (3.3) satisfy (3.4). It follows that the Krein extension of $J_{0}$ will also satisfy (3.4).

\section{REFERENCES}

1. M. G. Crandall and R. S. Phillips, On the extension problem for dissipative operators, J. Functional Analysis 2 (1968), 147-176.

2. M. A. Krein, The theory of self-adjoint extensions of semi-bounded Hermitian transformations and its applications. I, Mat. Sb. 20 (62) (1947), 431-495. (Russian)

3. R. S. Phillips, Dissipative operators and hyperbolic systems of partial differential equations, Trans. Amer. Math. Soc. 90 (1959), 193-254.

4. - On dissipative operators, Van Nostrand Math. Studies Series (to appear).

5. - The extension of dual subspaces invariant under an algebra, Proc. International Sympos. on Linear Spaces, Israel, (1960), Jerusalem Academic Press, 1961, pp. 366-398.

STANFORD UNIVERSITY 